\title{
Blunted Autonomic Reactivity to Mental Stress in Depression Quantified by Nonlinear Cardiorespiratory Coupling Indices
}

\author{
Spyridon Kontaxis ${ }^{1,2,3}$, Pablo Laguna ${ }^{1,2,3}$, Esther García ${ }^{3,4}$, Mar Posadas-de Miguel ${ }^{2}$, Sara Siddi ${ }^{5}$, \\ Maria Luisa Bernal ${ }^{2}$, Josep Maria Haro ${ }^{5,6}$, Jordi Aguiló ${ }^{3,4}$, Concepción de la Camara ${ }^{2,5}$, \\ Raquel Bailón ${ }^{1,2,3}$, and Eduardo Gil $1^{1,2,3}$ \\ ${ }^{1}$ BSICoS Group, I3A, University of Zaragoza, Spain \\ ${ }^{2}$ Instituto de Investigación Sanitaria de Aragón (IIS Aragón), Zaragoza, Spain \\ ${ }^{3}$ CIBER de Bioingeniería, Biomateriales y Nanomedicina, Spain \\ ${ }^{4}$ Microelectronics and Electronic Systems Dept, Autonomous University of Barcelona, Spain \\ ${ }^{5}$ CIBER de Salud Mental, Spain \\ ${ }^{6}$ Parc Sanitari Sant Joan de Déu, Sant Boi de Llobregat, Barcelona, Spain
}

\begin{abstract}
In this study, differences in autonomic reactivity to mental stress between Major Depressive Disorder (MDD) patients and healthy control (HC) subjects are assessed by nonlinear cardiorespiratory coupling indices derived from the Real Wavelet Biphase. The degree and strength of Quadratic Phase Coupling (QPC) between interacting oscillations of Heart Rate Variability $(H R V)$ and respiration are quantified before, during and after the execution of a cognitive task. Results show that the QPC strength and QPC degree between the respiration and the respiratory sinus arrhythmia component of HRV were lower in $\mathrm{HC}$ compared to MDD during stress, suggesting that the parasympathetic branch was less inhibited in MDD patients. During recovery, only in HC group, this degree of QPC increased, while the respiratory rate was reduced, compared to the basal stage. The degree of QPC between the respiration and components of $H R V$ in the low frequency band $([0.04,0.15] \mathrm{Hz})$ increased in HC during stress, compared to the basal stage, while remained unchanged in MDD patients. These results imply that depression is associated with blunted autonomic reactivity to mental stress.
\end{abstract}

\section{Introduction}

Major Depressive Disorder (MDD) is a mental health condition that imposes a heavy burden on people suffering from it [1]. One of the most common pathophysiological mechanisms underlying MDD is the imbalance of the Autonomic Nervous System (ANS) [2]. Differences in ANS regulation between healthy control (HC) subjects and depressed patients usually can be quantified by heart rate variability $(\mathrm{HRV})$ indices.

In a recent review, it was reported that MDD patients show lower levels of resting HRV as well as less executive functioning and emotion regulation during stress [3]. Besides HRV, respiratory activity has emerged as an objective measure of mood regulation since the action of the diaphragm can be altered by emotional states such as sadness and anxiety [4]. During sad mood, individuals with remitted depression showed greater respiration pattern variability compared to HC subjects [5].

Joint analyses of respiration and HRV may yield additional information on ANS functioning. Mutual information between HRV and respiration signals of healthy subjects showed high specificity in emotion recognition [6]. In [7], the inclusion of respiration in a HRV analysis improved the stress level quantification in healthy subjects. In [8], the analysis of linear cardiorespiratory interactions showed that MDD subjects, compared to HC, exhibit lower suppression of parasympathetic activity during stress.

A variety of studies suggest that the cardiorespiratory system exhibits nonlinear interactions which can be quantified by entropy [9] and phase [10] analyses, among others. One of the very few studies involving MDD patients, showed that entropy measures of cardiorespiratory coupling tend to increase as depression severity increases [9]. In this study, the nonlinear properties of the cardiorespiratory system are assessed with the Real Wavelet Biphase (RWB) [11], which quantifies the timephase relationship among oscillations of HRV and respiration that interact through a quadratic nonlinear system. The study of the nonlinear cardiorespiratory coupling function during ANS changes induced by mental stress might add clinical value to the diagnosis of MDD patients. 


\section{Dataset and Mental Stress Protocol}

A database of 40 MDD patients (24 women, age $45.4 \pm 13.3$ years, body mass index (BMI) $27.14 \pm$ $5.16 \mathrm{Kg} / \mathrm{m}^{2}$ ) with clinically significant depression and 40 age, sex, and BMI-matched healthy control (HC) subjects was recorded at the Hospital Clínico Lozano Blesa (Zaragoza, Spain) and the Mental Health Unit of the Parc Sanitari Sant Joan de Deú (Barcelona, Spain) under clinical studies PI16-0156 and PIC-148-16, respectively.

The experimental protocol is composed of three stages: (a) the basal stage $(\mathcal{B})$, where the subjects are filling psychometric tests [12], (b) the stressful stage $(\mathcal{S})$, where the subjects are performing the Trail Making Test, which consists in a non-verbal cognitive stressor [13], and (c) the recovery stage $(\mathcal{R})$, where the subjects are requested to relax during 5 minutes after the execution of the cognitive test.

Three orthogonal ECG leads and a respiratory signal (thoracic belt) were continuously recorded using Medicom system (ABP10 module of Medicom MTD, Ltd, Russia) at a sampling frequency of $1000 \mathrm{~Hz}$ and $250 \mathrm{~Hz}$, respectively.

\section{Nonlinear Cardiorespiratory Couplings}

The HRV signal was generated from the beat occurrence time series based on the integral pulse frequency modulation model and it was corrected by the mean heart rate [14]. Both HRV and respiration were resampled at $f_{s}=4 \mathrm{~Hz}$ and they were band-pass filtered in the range $[0.04,0.8] \mathrm{Hz}$.

When oscillations of two signals interact through a nonlinear system new harmonics with higher-order frequency and phase correlations will appear (Fig. 1).

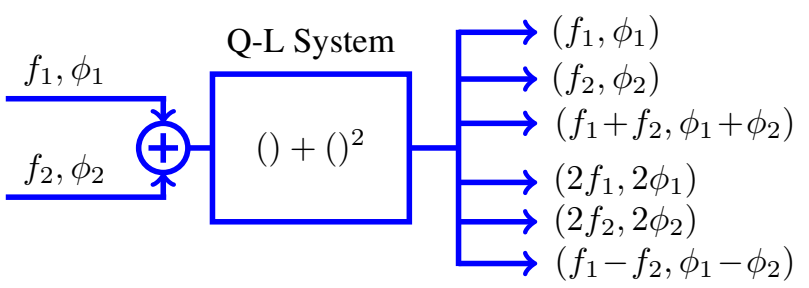

Figure 1. The harmonics generated by 2 interacting oscillations through a quadratic-linear (Q-L) system.

To study the time-varying cross-phase relationship between oscillations of HRV $(x(t))$ and respiration $(y(t))$, first, their Continuous Wavelet Transform (CWT) coefficients $W_{s}(f, t)=A_{s}(f, t) e^{j \phi_{s}(f, t)}, s \in\{x, y\}$ are computed in an interval of length $T$ sec, and then the instantaneous wavelet biphase $\Phi_{\mathrm{W}}\left(f_{1}, f_{2}, t\right)$ is obtained from,

$$
\Phi_{\mathrm{W}}\left(f_{1}, f_{2}, t\right)=\phi_{x}\left(f_{1}, t\right)+\phi_{y}\left(f_{2}, t\right)-\phi_{x}\left(f_{1}+f_{2}, t\right) .
$$

If the oscillations at frequencies $f_{1}$ and $f_{2}$ of $x(t)$ and $y(t)$, respectively, interact through a quadratic-linear system new harmonics will appear at $f_{1}+f_{2}$ with Quadratic Phase Coupling (QPC), i.e, $\phi_{x}\left(f_{1}+f_{2}, t\right)=\phi_{x}\left(f_{1}, t\right)+\phi_{y}\left(f_{2}, t\right)$. The QPC implies that $\Phi_{\mathrm{W}}\left(f_{1}, f_{2}, t\right)=0$ and it can be quantified with the bi-frequency domain of RWB,

$$
b_{\Phi}\left(f_{1}, f_{2}, t\right)=\frac{1}{T^{\prime}} \int_{t-T^{\prime} / 2}^{t+T^{\prime} / 2} \cos \left(\Phi_{\mathrm{W}}\left(f_{1}, f_{2}, \tau\right)\right) d \tau,
$$

where RWB is the mean cosine value of $\Phi_{\mathrm{W}}\left(f_{1}, f_{2}, t\right)$ around the central $T^{\prime} \mathrm{sec}$ of the analyzed interval $\left(T^{\prime}<T\right)$. High values of RWB imply that $\Phi_{\mathrm{W}}\left(f_{1}, f_{2}, t\right)$ is very small during the whole analyzed interval $T^{\prime}$, indicating a longerlasting phase synchronization. Surrogate data analysis is employed to obtain a statistically significant QPC threshold [11]. RWB values that do not exceed this threshold are set to zero. The same holds for values whose coordinates exceed half the intrinsic sampling frequency of HRV, which is given by the heart rate, since spectral components above this frequency are due to spectrum repetitions.

The regions where QPC will be assessed are defined based on respiratory rate information. The respiratory rate $f_{r}(t)$ is derived from $y(t)$ using the method proposed in [15]. QPC between the respiration and the respiratory sinus arrhythmia (RSA) component of HRV is identified in the region $\Omega_{R, R}:\left\{f_{1} \in \Omega_{R}, f_{2} \in \Omega_{R}\right\}$, where $\Omega_{\mathrm{R}}=\left[f_{r}(t)-0.05, f_{r}(t)+0.05\right] \mathrm{Hz}$, while QPC between respiration and low frequency components of HRV is identified in the region $\Omega_{\mathrm{L}, \mathrm{R}}:\left\{f_{1} \in \Omega_{\mathrm{L}}, f_{2} \in \Omega_{\mathrm{R}}\right\}$, where $\Omega_{\mathrm{L}}=[0.04,0.15] \mathrm{Hz}$. The involved frequencies to QPC, i.e, when $b_{\Phi}\left(f_{1}, f_{2}, t\right)>0$ are given by,

$$
\left(\hat{f}_{1}^{\mathcal{I}}, \hat{f}_{2}^{\mathcal{I}}\right)=\underset{f_{1}, f_{2} \in \Omega_{\mathcal{I}}}{\arg \max }\left\{b_{\Phi}\left(f_{1}, f_{2}, t\right)\right\}
$$

where $\mathcal{I}=\{[\mathrm{L}, \mathrm{R}],[\mathrm{R}, \mathrm{R}]\}$; for the simplicity of notation the dependence of $\hat{f}_{1}^{\mathcal{I}}, \hat{f}_{2}^{\mathcal{I}}$ on $t$ is omitted. The degree of QPC between the interacting frequencies is quantified with,

$$
C_{\mathcal{I}}^{\Phi}(t)=b_{\Phi}^{2}\left(\hat{f}_{1}^{\mathcal{I}}, \hat{f}_{2}^{\mathcal{I}}, t\right)
$$

where lower values imply that the phase locking in the involving frequencies is reduced, i.e., $\Phi_{\mathrm{W}}\left(f_{1}, f_{2}, t\right) \neq 0$. The strength of QPC is assessed with the Normalized Wavelet Biamplitude (NWB):

$$
E_{\mathcal{I}}(t)=\frac{\left|\int_{t-T^{\prime} / 2}^{t+T^{\prime} / 2} A_{\mathrm{W}}\left(\hat{f}_{1}^{\mathcal{I}}, \hat{f}_{2}^{\mathcal{I}}, \tau\right) e^{j \Phi_{\mathrm{W}}\left(\hat{f}_{1}^{\mathcal{I}}, \hat{f}_{2}^{\mathcal{I}}, \tau\right)} d \tau\right|}{\int_{t-T^{\prime} / 2}^{t+T^{\prime} / 2}\left|W_{x}\left(\hat{f}_{1}^{\mathcal{I}}, \tau\right) W_{y}\left(\hat{f}_{2}^{\mathcal{I}}, \tau\right)\right| d \tau}
$$

where $A_{\mathrm{W}}\left(f_{1}, f_{2}, t\right)=A_{x}\left(f_{1}, t\right) A_{y}\left(f_{2}, t\right) A_{x}\left(f_{1}+f_{2}, t\right)$ is the instantaneous wavelet biamplitude from which the amplitude influence of the interacting oscillations (denominator) is removed. Thus, NWB is influenced only by the 
coupling strength and the amplitude of the nonlinear oscillations in $\operatorname{HRV}\left(A_{x}\left(f_{1}+f_{2}, t\right)\right)$.

The parameters $C_{\mathcal{I}}^{\Phi}(t)$ and $E_{\mathcal{I}}(t)$ are estimated every $10 \operatorname{secs}\left(T=60 \mathrm{sec}, T^{\prime}=10 \mathrm{sec}\right)$ and only when $f_{r}(t)>0.15 \mathrm{~Hz}$. The time percentage of QPC in $\Omega_{\mathrm{R}, \mathrm{R}}\left(\mathcal{T}_{\mathrm{R}, \mathrm{R}}\right)$ and $\Omega_{\mathrm{L}, \mathrm{R}}\left(\mathcal{T}_{\mathrm{L}, \mathrm{R}}\right)$ is also calculated; further details can be found in [11]. An example of QPC quantification is shown in Fig. 2.

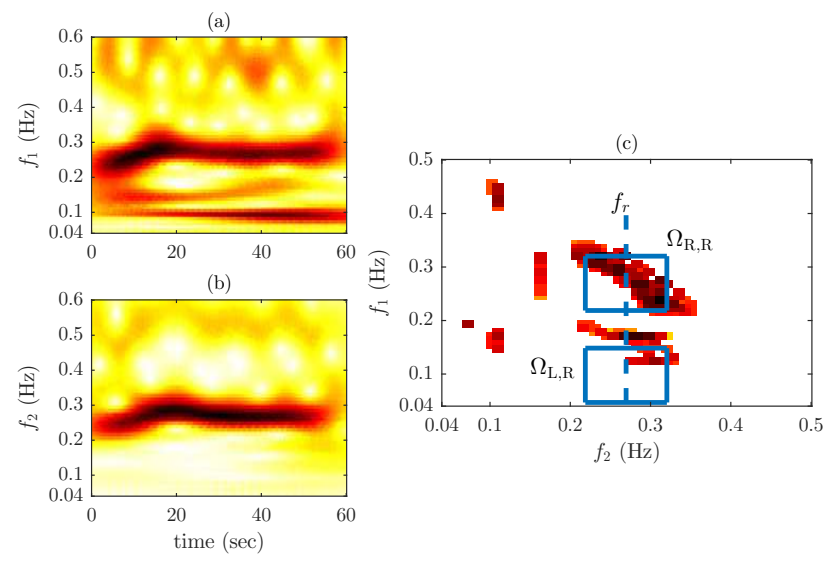

Figure 2. QPC assessment in the regions related to respiration. The CWT amplitude coefficients of (a) $\mathrm{HRV}\left|W_{x}\left(f_{1}, t\right)\right|$, (b) respiration $\left|W_{y}\left(f_{2}, t\right)\right|$, and (c) the cross-RWB $b_{\Phi}\left(f_{1}, f_{2}, t=30 \mathrm{sec}\right)$. The respiratory rate $f_{r}$ is marked with a dashed blue line, while the regions $\Omega_{\mathrm{L}, \mathrm{R}}$ and $\Omega_{\mathrm{L}, \mathrm{R}}$ are marked with solid blue lines.

\subsection{Statistical Analysis}

The median value of each feature, $C_{\mathrm{L}, \mathrm{R}}^{\Phi}, C_{\mathrm{R}, \mathrm{R}}^{\Phi}, E_{\mathrm{L}, \mathrm{R}}$, $E_{\mathrm{R}, \mathrm{R}}$, and $f_{r}$, is obtained for each subject and stage. Individual differences are assessed separately for $\mathrm{HC}$ and MDD group. A multicomparison test with Bonferroni correction $(N=2)$ is carried out for evaluating the individual differences at $\mathcal{S}$ and $\mathcal{R}$ stages with respect to $\mathcal{B}$ using the paired t-test, and the Wilcoxon signed rank test when appropriate. Differences in ANS regulation between MDD and $\mathrm{HC}$ group are assessed with the t-test for independent samples, and the Mann-Whitney U non-parametric test when appropriate. The significance threshold in this study is set to $p<0.025$ (Bonferroni correction $N=2$ ).

\section{Results}

The time percentages (mean \pm standard deviation) where QPC was present in $\Omega_{R, R}$ and $\Omega_{L, R}$ are illustrated in Table 1 . No significant differences were found for both $\mathcal{T}_{\mathrm{R}, \mathrm{R}}$ and $\mathcal{T}_{\mathrm{L}, \mathrm{R}}$ neither when evaluating the individual differences nor for the group differences.
Table 1. Time percentage of QPC (mean \pm std $)$

\begin{tabular}{c|cc|cc|}
\cline { 2 - 5 } & \multicolumn{2}{c}{$\mathrm{HC}$} & \multicolumn{2}{c}{ MDD } \\
\hline $\mathcal{B}$ & $31.8 \pm 24.5$ & $39.4 \pm 17.2$ & $37.5 \pm 23.1$ & $38.7 \pm 17.8$ \\
$\mathcal{S}$ & $33.7 \pm 24.9$ & $37.1 \pm 22.5$ & $37.1 \pm 25.2$ & $44.9 \pm 22.8$ \\
$\mathcal{R}$ & $35.1 \pm 26.2$ & $37.6 \pm 21.7$ & $38.9 \pm 25.0$ & $43.7 \pm 19.1$ \\
\hline
\end{tabular}

Boxplots of the features are shown in Fig. 3. Results show that the QPC strength, $E_{\mathrm{R}, \mathrm{R}}$, and the QPC degree, $C_{\mathrm{R}, \mathrm{R}}^{\Phi}$, (Fig. 3(a), (c)) are statistically significantly higher in MDD compared to HC subjects during stress $(\mathcal{S})$. In the HC group, stress induced by the cognitive task execution increased the degree of QPC in $\Omega_{\mathrm{L}, \mathrm{R}}$ compared to basal stage $\mathcal{B}$ (Fig. 3(d)). Furthermore, in HC group, the QPC degree $C_{\mathrm{R}, \mathrm{R}}^{\Phi}$ increased after task execution, while the respiratory rate $f_{r}$ decreased significantly compared to $\mathcal{B}$ (Fig. 3(c), (e)). No significant intra-subject differences along the protocol stages were found in MDD group.

\section{Discussion}

In this study differences in ANS regulation induced by a cognitive stressor between MDD and healthy subjects are assessed with the degree and strength of QPC between oscillations of HRV and respiration.

Results show that the QPC strength, $E_{\mathrm{R}, \mathrm{R}}$, and the QPC degree, $C_{\mathrm{R}, \mathrm{R}}^{\Phi}$, between the respiration and the RSA component of HRV were statistically significantly lower in HC compared to MDD subjects during stress (Fig. 3(a), (c)). This suggests that the parasympathetic branch was less inhibited in MDD patients, which was also reported in [8], wherein HC compared to MDD subjects showed reduced linear cardiorespiratory coupling during stress. Moreover, a vagal withdrawal induced by a tilt-table test resulted to a decreased QPC strength in $\Omega_{\mathrm{R}, \mathrm{R}}$ as well as in $\Omega_{\mathrm{L}, \mathrm{R}}$ [11].

An increased QPC degree $C_{\mathrm{L}, \mathrm{R}}^{\Phi}$ during stress $\mathcal{S}$ compared to basal $\mathcal{B}$ was found in $\mathrm{HC}$ subjects (Fig. 3(d)). This might be associated with an increased phase synchronization between respiration and LF components of HRV during stress. Higher QPC degree implies higher phase synchronization between the interacting components. QPC strength depends on the amplitude of the nonlinear oscillations in HRV that might be reduced during stress, and on QPC degree [11]. If biphase is maintained constant, $E_{\mathcal{I}}(t)$ will get higher values. Thus, a higher degree of QPC during the analyzed period could attenuate the reduction in QPC strength $E_{\mathrm{L}, \mathrm{R}}$ during stress and it may explain the absence of significant differences in the HC group.

Moreover, during the recovery stage $\mathcal{R}$, the QPC strength, $E_{\mathrm{R}, \mathrm{R}}$, increased significantly compared to basal stage $\mathcal{B}$ for HC group (Fig. 3(c)). This suggests an increase of parasympathetic activity. As can been seen from Fig. 3(e), the expected sympathetic inhibition in healthy 

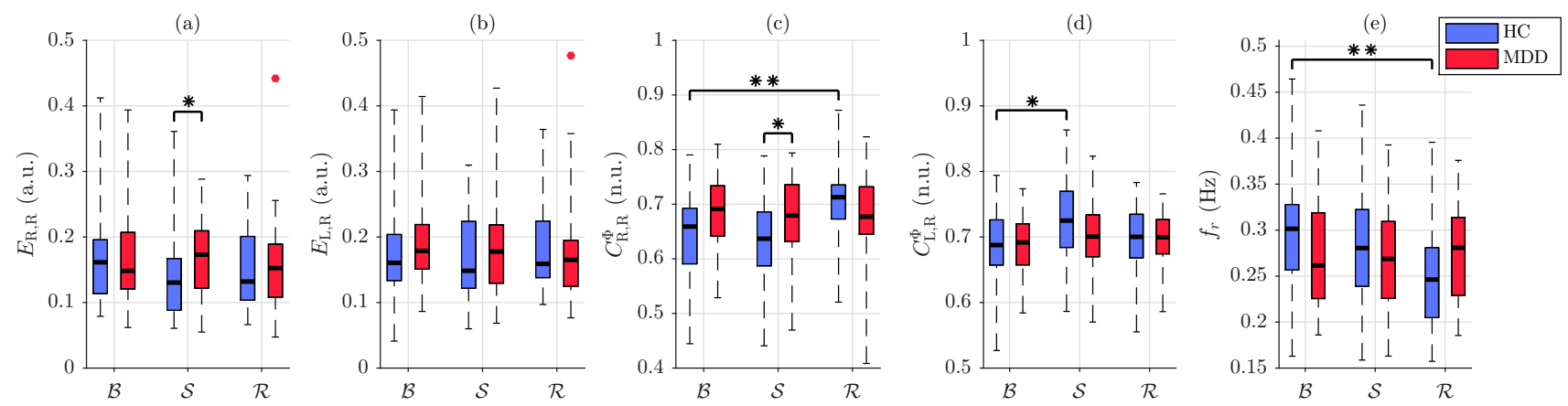

Figure 3. Boxplots of the features. The QPC strength (a) $E_{\mathrm{R}, \mathrm{R}}$, and (b) $E_{\mathrm{L}, \mathrm{R}}$, the QPC degree (c) $C_{\mathrm{R}, \mathrm{R}}^{\Phi}$, and (d) $C_{\mathrm{L}, \mathrm{R}}^{\Phi}$, and, (e) the respiratory rate $f_{r}$. The group of HC and MDD subjects are marked in blue and red, respectively. Statistical significant differences are marked with one asterisk when $p<0.025$ and with two asterisks when $p<0.001$.

subjects during $\mathcal{R}$ is associated with a lower respiratory rate $f_{r}$ compared to $\mathcal{B}$, while the respiratory rate remained unchanged in MDD patients. In general, no significant intra-subject differences along the protocol stages were found in MDD group. These results support blunted autonomic reactivity to mental stress in MDD subjects, which are in agreement with previous studies [3,8], where parasympathetic branch in MDD patients was found to be less inhibited during mental stress. In [16], higher degree of depression was associated with a decreased autonomic reactivity, thereby highlighting the significance of stress response quantification in depression.

\section{Conclusions}

Nonlinear cardiorespiratory coupling indices, including the degree and strength of Quadratic Phase Coupling, show that MDD patients exhibit a blunted autonomic reactivity to mental stress. The relationship among interacting oscillations of $\mathrm{HRV}$ and respiration with quadratic nature, associated with the nonlinear cardiorespiratory coupling function, might add clinical value to the depression diagnosis.

\section{Acknowledgment}

This work was supported under the project RTI2018-097723-B-I00 funded by AEI and FEDER, LMP44-18 by Aragón Government (AG) and FEDER, AG with a personal grant to S. Kontaxis, European Social Fund through BSICoS group (T39_20R). The computation was performed by the ICTS "NANBIOSIS", more specifically by the High Performance Computing Unit of the CIBER in Bioengineering, Biomaterials \& Nanomedicine (CIBER-BBN) at the University of Zaragoza.

\section{References}

[1] James SL, et al. Global, regional, and national incidence, prevalence, and years lived with disability for 354 diseases and injuries for 195 countries and territories, 1990-2017: a systematic analysis for the Global Burden of Disease Study 2017. The Lancet 2018; 392(10159):1789-1858.

[2] Angermann CE, Ertl G. Depression, anxiety, and cognitive impairment. Curr Heart Fail Rep 2018;15(6):398-410.
[3] Schiweck C, et al. Heart rate and high frequency heart rate variability during stress as biomarker for clinical depression. a systematic review. Psychol Med 2019;49(2):200-211.

[4] Bordoni B, et al. A review of analgesic and emotive breathing: a multidisciplinary approach. J Multidiscip Healthc 2016;9:97.

[5] Zamoscik VE, et al. Respiration pattern variability and related default mode network connectivity are altered in remitted depression. Psychol Med 2018;48(14):2364-2374.

[6] Valderas MT, et al. Mutual information between heart rate variability and respiration for emotion characterization. Psychol Meas 2019;40(8):084001.

[7] Hernando A, et al. Inclusion of respiratory frequency information in heart rate variability analysis for stress assessment. IEEE J Biomed Health Inform 2016;20(4):1016-1025.

[8] Kontaxis S, et al. Heart rate variability analysis guided by respiration in major depressive disorder. In 2018 Computing in Cardiology Conference (CinC), volume 45. IEEE, 2018; 1-4.

[9] Zhao L, et al. Cardiorespiratory coupling analysis based on entropy and cross-entropy in distinguishing different depression stages. Front Physiol 2019;10:359.

[10] Kontaxis S, et al. Mental stress detection using cardiorespiratory wavelet cross-bispectrum. In 2016 Computing in Cardiology Conference (CinC). IEEE, 2016; 725-728.

[11] Kontaxis S, et al. Assessment of quadratic nonlinear cardiorespiratory couplings during tilt-table test by means of real wavelet biphase. IEEE Trans Biomed Eng 2018;66(1):187-198.

[12] Arza A, et al. Measuring acute stress response through physiological signals: towards a quantitative assessment of stress. Med Biol Eng Comput 2019;57(1):271-287.

[13] McMorris T. Chapter 1 - History of Research into the Acute Exercise-Cognition Interaction: A Cognitive Psychology Approach. In McMorris T (ed.), Exercise-Cognition Interaction. San Diego: Academic Press, 2016; 1 - 28.

[14] Bailón R, et al. The integral pulse frequency modulation model with time-varying threshold: application to heart rate variability analysis during exercise stress testing. IEEE Trans Biomed Eng 2010;58(3):642-652.

[15] Kontaxis S, et al. ECG-derived respiratory rate in atrial fibrillation. IEEE Trans Biomed Eng 2020;67:905-914.

[16] Kontaxis S, et al. Photoplethysmographic waveform analysis for autonomic reactivity assessment in depression. IEEE Trans Biomed Eng 2020.

Address for correspondence:

Spyridon Kontaxis (sikontax@unizar.es)

Campus Río Ebro, C/ María de Luna 1, 50018 Zaragoza, Spain 